\title{
Application of Robust Fixed Point control in case of T1DM
}

\author{
György Eigner*, Péter Horváth ${ }^{\dagger}$, József K. Tar ${ }^{\ddagger}$, Imre J. Rudas ${ }^{\ddagger}$, and Levente Kovács* \\ ${ }^{*}$ Research and Innovation Center of Obuda University, \\ Physiological Controls Group, Obuda University, Budapest, Hungary \\ Email: eigner.gyorgy@phd.uni-obuda.hu, kovacs.levente@nik.uni-obuda.hu \\ ${ }^{\dagger}$ Department of Control Engineering and Information Technology, \\ Budapest University of Technology and Economics, Budapest, Hungary \\ Email: peter.87.horvath@gmail.com \\ ${ }^{\ddagger}$ Research and Innovation Center of Obuda University, \\ Antal Bejczy Center for Intelligent Robotics, Obuda University, Budapest, Hungary \\ Email: tar.jozsef@nik.uni-obuda.hu,rudas@uni-obuda.hu
}

\begin{abstract}
Adaptive, model-free control of Type 1 Diabetes Mellitus (T1DM) is a lack in the field of diabetes control, since, most of the applied control strategies are model-based ones. The main problem is that difficult to formulate exact mathematical models to replicate the physiological processes, not just because of their behavior, rather then these processes are changing patient-by-patient. Furthermore, the developed models so far, are highly non-linear and difficult to manage. A possible adaptive control solution can be the recently developed Robust Fixed Point Transformation (RFPT)-based control design method, which can provide control action, based on the observations about the actual output of a controlled system. In this paper we show a survey, how can be used this novel technique related with a known, highorder glucose-insulin model, to investigate the usability according to diabetes control.
\end{abstract}

Index Terms-Robust Fixed-Point Transformation, RFPT, T1DM, Control of Diabetes

\section{INTRODUCTION}

Diabetes Mellitus (DM) is a sub-group of the so-called Chronic Metabolic Diseases (CMD), connected to the insulin hormone, which is the key compound of the glucose uptake by several types of body cells [1]. Glucose, beside other low complex carbohydrates $(\mathrm{CHO}(\mathrm{s}))$ provides the energy for most of the human body cells, among others, through the Citric-Acid cycle.

The most dangerous type of DM is Type 1 DM (T1DM), which occurs when the insulin producer $\beta$-cells of the pancreas are annihilated by an autoimmune reaction. Hence, because of the lack of insulin, the insulin-dependent cells of the human body are going to be suffer energetic deficit on short term and energetic collapse over time [2].

Modeling and control have absolute relevance on the diabetes research field. The main problems are associated with the fact that the physiological processes in human body are non-linear, thus, the control design is not trivial and demands individual approach case-by-case [3].

Taking into account that it is very difficult to precisely modeling and identifying any physiological processes, nonlin- ear, robust or adaptive control solution can provide appropriate control action based on approximate model of the real system. One of the most common non-linear technique is based on Lyapunov's 2nd law [4], but this is difficult to handle mathematically. Frequently used non-linear techniques are the Nonlinear Model Predictive Control (NMPC) [5], Soft Computing techniques [6], [7], and Linear Parameter Varying (LPV) based robust control methods [8]-[10]. Adaptive controllers can nicely adapt themselves to need of the system under control by observing its behavior, like the Model Reference Adaptive Control (MRAC) [11].

In this paper we investigate how the recently developed RFPT based control design can give a useful solution to diabetes control [12], [13]. This method has several benefits that will be detailed below.

The paper is structured as follows. First, we give a short introduction about the handled problem. Following that we present the investigated T1DM model and the control method applied to it. At last, we summarize our results, draw the conclusions, and outline a perspective for the future work.

\section{T1DM MODEL}

During the investigations, we used a well-known, high order digestion (1) and T1DM (2) models, presented by [14]-[17]. Also, this models are the base of the UVA/Padova simulator [18]. The model schematic figure are represented by Fig. 1.

$$
\begin{aligned}
& \dot{Q}_{s t o}(t)=Q_{s t o 1}(t)+Q_{s t o 2}(t) \\
& \dot{Q}_{\text {sto } 1}(t)=-k_{\text {gri }} Q_{\text {sto } 1}(t)+d(t) \\
& \dot{Q}_{\text {sto } 2}(t)=-k_{\text {gut }}\left(t, Q_{\text {sto }}\right) Q_{\text {sto } 2}(t)+k_{\text {gri }} Q_{\text {sto } 1}(t) \\
& \dot{Q}_{\text {gut }}(t)=-k_{\text {abs }} Q_{\text {gut }}(t)+k_{\text {gut }}\left(t, Q_{\text {sto }}\right) Q_{\text {sto } 2}(t) \\
& R a(t)=\frac{f k_{a b s} Q_{g u t}(t)}{B W} \text {. }
\end{aligned}
$$




$$
\begin{aligned}
& \dot{G}_{M}(t)=-k_{s c} G_{M}(t)+\frac{k_{s c}}{V_{G}} G_{p}(t) \\
& \dot{G}_{p}(t)=E G P(t)+R a(t)-U_{i i}-E(t)- \\
& \quad-k_{1} G_{p}(t)+k_{2} G_{t}(t) \\
& \dot{G}_{t}(t)=-U_{i d}+k_{1} G_{p}(t)-k_{2} G_{t}(t) \\
& \dot{X}_{t}(t)=-p_{2 U} X(t)+p_{2 U}\left[I(t)-I_{b}\right] \\
& \dot{I}_{d}(t)=-k_{i} I_{d}(t)+k_{i} I_{1}(t) \\
& \dot{I}_{1}(t)=-k_{i} I_{1}(t)+k_{i} I(t) \quad m_{1} I_{l}(t)+k_{a 2} S_{2}(t)+ \\
& \dot{I}_{p}(t)=-\left(m_{2}+m_{4}\right) I_{p}(t)+k_{a 1} S_{1}(t) \\
& \dot{I}_{l}(t)=m_{2} I_{p}(t)-\left(m_{1}+m_{3}\right) I_{l}(t) \\
& \dot{S}_{2}(t)=-k_{a 2} S_{2}(t)+k_{d} S_{1}(t) \\
& \dot{S}_{1}(t)=-\left(k_{a 1}+k_{d}\right) S_{1}(t)+u(t)
\end{aligned}
$$

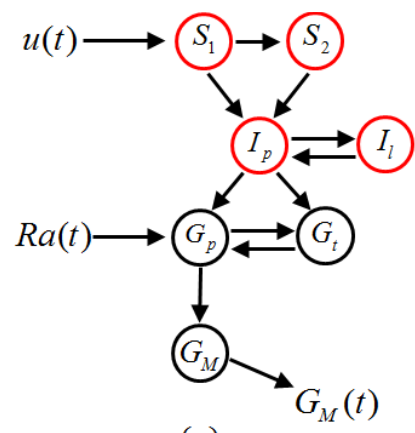

(a)

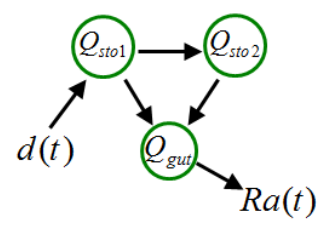

(b)

Figure 1. The schematic structures of the used models [( $a$ ) High-order T1DM model, (b) Complex digestion model]

The unified complex model has two inputs and one output, namely, $u(t)[\mathrm{pmol} / \mathrm{kg} / \mathrm{min}]$ is the injected insulin, $d$ $[\mathrm{mg} / \mathrm{min}]$ is the amount of ingested glucose and $G_{M}(t)[\mathrm{mg} / \mathrm{dl}]$ represents the subcutaneous glucose level. Other details are available in the cited literature.

\section{Applied Control Method}

The basic idea of the RFPT-based adaptive control is the concept of the "response function" that belongs to the compound consisting of the "rough initial model in use" and the "actual physical system under control". For a purely kinematically formulated "desired response" of the controlled system $r^{D e s}$ the necessary control signal is designed by the use of the approximate model. By applying this control signal on the actual system, its realized response, $r^{\text {Real }}$, will be a function of $r^{\text {Des }}$ as $r^{\text {Real }}=f\left(r^{\text {Des }}\right) \neq r^{\text {Des }}$ due to the modeling errors and the unknown external disturbances. The task is to find the appropriate input $r_{\star}$ to which the situation $r^{\text {Des }}=f\left(r_{\star}\right)$ belongs. Normally $r_{\star}$ can be found as the limit of a sequence obtained by the iterative application of a contractive map as $r_{n+1}=G\left(r_{n} ; r^{D e s}\right)$ that is composed by the use of the response function $f$. (The discrete steps correspond to the digital cycles of the controller.) The RFPTbased design corresponds to giving a particular choice for the function $G$. The convergence of the so obtained sequence depends partly on the parameters of $G$ and partly on the behavior of $f$. It was shown that for a wide class of physical systems this convergence can be guaranteed by properly setting the parameters of $G$ if $\frac{\partial f}{\partial r}$ satisfies some simple requirements [19]. The mathematical background corresponds to Banach's "Fixed Point Theorem" [20] that states that the limit value of an iterative sequence $\left\{x_{n+1}=G\left(x_{n}\right)\right\}$ generated a contractive map $G: \Re^{n} \mapsto \Re^{n}$ on a linear, normed, complete metric space (Banach space) is the fixed point of $G$ satisfying the equation $G\left(x_{\star}\right)=x_{\star}$. For Single Input - Single Output (SISO) systems

$$
\begin{gathered}
r_{n+1}=G\left(r_{n} ; r^{\text {Des }}\right) \stackrel{\text { def }}{=}\left(r_{n}+K_{c}\right) \times \\
\left\{1+B_{c}\left[\tanh \left(A_{c}\left(f\left(r_{n}\right)-r^{D e s}\right)\right)\right]\right\}-K_{c}
\end{gathered}
$$

where $K_{c}, A_{c}$, and $B_{c}= \pm 1$ are the adaptive control parameters. Evidently we have two fixed points as $r=-K_{c}$ (that is trivial and cannot used in the control), and $r_{\star}$ for which $f\left(r_{\star}\right)=r^{D e s}$, that is the solution of the control task. For convergence the $\left|\frac{\mathrm{d} f}{\mathrm{~d} r}\right|<1$ condition must be met. Further explanation on appropriate setting the adaptive control parameters can be found in [13].

\section{Controller Design}

\section{A. Specifics of the Model}

During the development, quite a few general control, physiological and phenomenological constraints have to be considered, as listed below:

- With the caveats that each of the state variables denotes the concentration of certain chemical component, therefore ot must have either positive value, or after the depletion of the appropiate component- it must ramain zero. These "truncation-type" nonlinearities make the application of any "linearization" dubious, whenever a given component is depleted. This fact seriously concerns any considerations related to the frequency domain that can widely used in the case of linear more or less linearizable systems. In the sequel qualitative analysis of present model is given.

- Each time-constant model parameter should be positive.

- Each state variable has its own exponential decay constant.

- Any coupling between the coupled pairs of state variables is of exponential nature: the decrease in the concentration of given state variable generates increase in that of the coupled one and vice-versa.

- Without finite input or extraction terms all the state variables should converge to zero. 
- The state propagation quantities as $\dot{X}, \dot{I}_{d}, \dot{I}_{1}, \dot{I}_{p}, \dot{I}_{L}, \dot{S}_{2}$ and $\dot{S}_{1}$ are completely independent of the state variables $G_{M}, G_{p}$ and $G_{t}$.

- Each element of the state propagation group $\dot{G}_{M}, \dot{G}_{p}$ and $\dot{G}_{t}$ directly is concerned by the input $\dot{R}_{a}$ and the state variables $\mathrm{X}$ and $\dot{I}_{d}$ belonging to the other group.

- To sum up, $u>0$ makes $X$ increase. Increasing $X$ decrease $\dot{G}_{t}$ and $G_{t}$. Decreasing $G_{t}$ decreases $\dot{G}_{p}$ due to which (2) gives possibility for decreasing $G_{M}$ by properly big insulin input $\mathrm{u}$. It is important to note that depletion of $\mathrm{X}$-since $\operatorname{dot} X=0$ if $X=0$ - makes any possibility for controlling $G_{M}$ via u cease. This introduces a strong nonlinear asymmetry into the system: drastic glucose $R_{a}$ drastically increase $G_{M}$, via drastic insulin input u its effect can be contained.

- From 1 it is evident that $\mathrm{u}$ is directly related to a high order time-derivative of the directly measureable state variable $G_{M}$.

\section{B. The Effect chain of Control Action}

To design the Rough Model (RM), which provides the control signal, the effect chain of injected insulin (which is the control signal) need to be mapped. This route defines, how can we affect on the operation of the system, without knowledge of the actually happening inside the system.

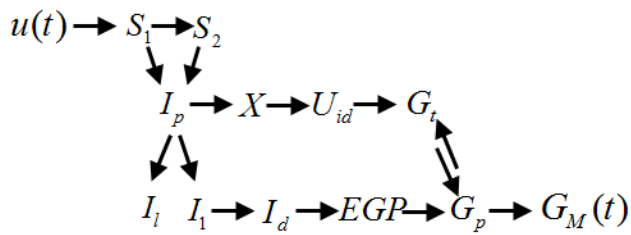

Figure 2. Effect chain of insulin

Fig. 2. shows the concrete effect chain of injected insulin $u(t)$. The physiological model specialties determine that how the injected insulin affects on the $G_{M}(t)$, namely, through the insulin-dependent glucose utilization $\left(U_{i d}\right)$, which is the glucose uptake by insulin-dependent tissues and the endogenous glucose production of the liver $(E G P)$, thus, the inhibition of gluconeogenesis.

\section{Design of Rough Model}

It can be concluded based on the IV-B that a kinematic prescription (which is used by the RFPT-method) are not expedient in this case, since, the relative order of the control chain is at least 8 . That means, the kinematic prescription should be contains the 8th time derivative of the $G_{M}$, at the same time, the required order of the control law should be 8 as well, in order to handle this high derivative. To avoid this unpleasant effect, other approaches should be used. A possible solution, if the exact model is "hided", as a black box and only the input and output are investigated. Naturally, this step is reducing the accuracy of the control, nevertheless, it can be used, because of the adaptivity of the method. If the steady state of the system can be approached over one cycle and the $G_{M}$ is available at the end of this cycle, the necessary insulin input which is need to be injected at the next cycle can be calculated by the controller. However, this condition is not usable itself, because of the glucose input dynamics is faster then the system's settling characteristics. Therefore, a simple dynamic scheme has to be developed, which can describes not only the $\Delta \tilde{u}$ determined by the actual glucose input, but also the takes into account the affect of the past control actions on the actual condition beside the appropriate time delays, determined by the system's dynamics. Here, we supposed that the $R_{a}$ is known, since the glucose input is regulated by us and based on the (1), the $\tilde{G_{M}}\left(\tilde{u} ; \tilde{R_{a}}\right)$ can be calculated. The selected equation is a quite simple one,

$$
G_{M}(t) \approx a u(t)+b
$$

Let $\tilde{u} \in[250,600]$ and $\tilde{G}_{M}$ is calculated, where $\tilde{R}_{a}=60 n$, where $n \in\{0 . .15\}$ integer. Based on (4), with numerical approximation, the $a$ and $b$ are calculable. To reduce complexity, a second order polynomials can be fitted on the $a\left(R_{a}\right)$ and $b\left(R_{a}\right)$ which occurred with the calculation of $a$ and $b$.
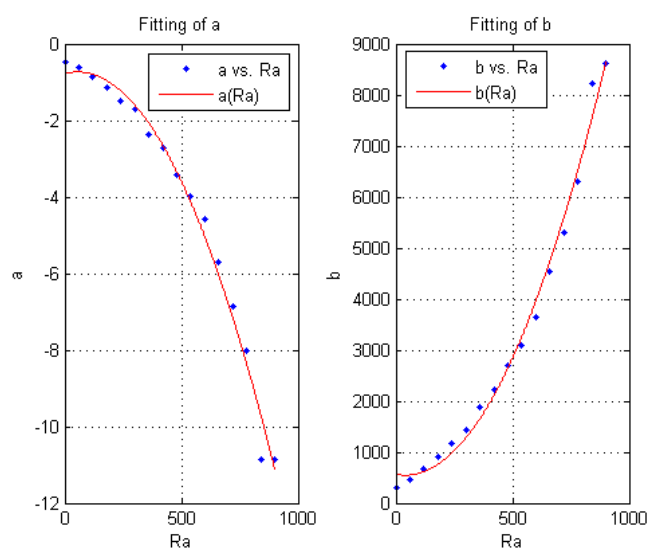

Figure 3. Results of curve fittings based on (4) (for example, beside $n=0$, $\tilde{R}_{a}=0, \tilde{u}_{\min }=250$ and $\left.\tilde{u}_{\max }=600\right)$, then $\tilde{G}_{M, s t a c}\left(u_{\min }\right) \approx 200$ and $\tilde{G}_{M, s t a c}\left(u_{\max }\right) \approx 40$, the numerical calculation gives $a_{e s t}=-0.46$ and $\left.b_{\text {est }}=314.29\right)$

Due to the fact, that the polynomials are known, $\Delta \tilde{u}$ can be estimated by:

$$
\begin{aligned}
\Delta G_{M}^{\text {Desired }}(t) \approx \frac{d a\left(R_{a}\right)}{d R_{a}} \Delta R_{a} \tilde{u} & +a\left(R_{a}\right) \Delta \tilde{u}+ \\
& +\frac{d b\left(R_{a}\right)}{d R_{a}} \Delta R_{a},
\end{aligned}
$$

where $\Delta \tilde{G}_{M}^{\text {Desired }}(t)$ denotes the changing of the desired subcutaneous glucose level determined by the measurements. The prescribed approximation for $\Delta \tilde{G}_{M}^{\text {Desired }}(t)$ is "purely" kinematic and contains several simplification from the control 
point of view. These simplifications are occurring as strong constraints during the control and the inaccuracies should be controlled by the adaptivity law.

The RM can be constructed with using the second order polynomials and combine with (1), (4) and (5):

$$
\begin{array}{r}
\dot{u}^{\text {Desired }}=\frac{G_{M}+\dot{G}_{M}^{\text {Desired }}-a\left(R_{a}\right) u-b\left(R_{a}\right)}{a\left(R_{a}\right)}- \\
-\frac{\frac{d a\left(R_{a}\right)}{d R_{a}} u-\frac{d b\left(R_{a}\right)}{d R_{a}}}{a\left(R_{a}\right)}
\end{array}
$$

The RM, according to the (6) gives an estimation about the $G_{M}$ and $u$ and provides the control signal as well. The tuning parameters are the specifics of the polynomials, $a\left(R_{a}\right)$ and $b\left(R_{a}\right)$. These approximation includes that physical constraint at the same time that the control signal, namely, the injected insulin cannot be negative. It can be seen, that finally, the changing of the desired insulin level is determined by the followings:

- Actual BG measurements $\left(G_{M}\right)$, which are available at every 5 minutes. We used this value, because the CGMS systems on the market are operating with this sampling time.

- The changing of the desired BG level $\left(\dot{G}_{M}\right)$, affected by the control law.

- The used polynomials and the changing of them, affected by the $u$. Furthermore, the polynomials are determined by the glucose input, which is a good approximation of the reality, where the insulin dosing is determined by the ingested food, namely, the glucose input.

\section{Control Law}

The control law can be formalized with the kinematic requirements. Due to the RM is a second order model, the control law should be a second order one as well. From simplicity reasons we implemented a "Fixed Set-Point type of Control" with $G^{N}$ as set-point parameter. The tracking error is taken as a prescription and such a PID kind feedback with a proportional term $\Lambda>0$ could be suitable:

$$
\left(\frac{\mathrm{d}}{\mathrm{d} t}+\Lambda\right)^{3} \int_{t_{0}}^{t_{1}}\left(G^{N}(\xi)-G(\xi)\right) d \xi=0
$$

where $G^{N}(t)$ is the nominal blood glucose concentration of the nominal model, $G(t)$ is the realized blood glucose concentration and the exact requirement is that the error signal, $G^{N}(t)-G(t)$, should converge to zero as $t \rightarrow \infty$. Naturally, the fixed-set point control determines that the derivatives of $G^{N}$ will be zero. With mathematical transformations of (7), the desired $G_{M}$ derivate is equal to

$$
\begin{aligned}
& \ddot{G}_{M}^{\text {Desired }}(t)=\left(\frac{\mathrm{d}}{\mathrm{d} t}\right)^{2} G^{N}(t)+ \\
& +\sum_{s=0}^{2}\left(\begin{array}{l}
3 \\
s
\end{array}\right) \Lambda^{3-s}\left(\frac{\mathrm{d}}{\mathrm{d} t}\right)^{s} \int_{t_{0}}^{t_{1}}\left(G^{N}(\xi)-G(\xi)\right) d \xi .
\end{aligned}
$$

where $G^{N}(t)$ is the BG concentration of the nominal model, $G(t)$ is the realized blood glucose concentration and the exact requirement is that the error signal, $G^{N}(t)-G(t)$, should converge to zero as $t \rightarrow \infty$. Naturally, the fixed-set point control determines that the derivatives of $G^{N}$ will be zero.

We implemented a "forgetting integral", because the former tracking errors were considered with lower weight to dismiss the overload of the integrated error. The changing of $\dot{G}_{M}^{D e s i r e d}$ is reflecting in the RM and affects the injected insulin level at every cycle.

\section{E. Adaptivity Law}

The suggested adaptivity law from [13] is the (3). We selected an other adaptivity law, which also satisfies the mathematical requirements of the RFPT-based method, which was the following:

$$
\begin{gathered}
r_{n+1}=G\left(r_{n} ; r^{\text {Des }}\right) \stackrel{\text { def }}{=}\left(r_{n}+K_{c}\right) \times \\
\left\{1+B_{c}\left[\Psi\left(A_{c}\left(f\left(r_{n}\right)-r^{D e s}\right)\right)\right]\right\}-K_{c}
\end{gathered}
$$

where $K_{c}, A_{c}$, and $B_{c}= \pm 1$ are the adaptive control parameters and $\Psi$ is a sigmoid function (similar properties as the hyperbolic tangent), namely $\Psi(x)=\frac{x}{1+|x|}$.

\section{F. Control scheme}

Fig. 4. shows the schematic control structure of the system. The RFPT-based methodology is clearly visible on this figure, thus, the control action is based on the known input and the measurable output of the system and the controller does not have knowledge about the inside conditions of the controlled system. This philosophy has absolute relevance for the physiological systems, where exact knowledge about the inside operation of the controlled system is not available.

\section{G. Constraints of Usability}

In this given case, several constraints need to be considered which come from the used models and adapted control scheme, detailed below.

1) Constraints about the used models: The selected patient model is a high order and complex one, with different inside dynamics at glucose and insulin subsystems. The insulin affects on the glucose subsystem through a complex route. We implemented the model with the data given by [14]-[17], without identification, which take in further uncertainty to the system. We have considered, that the input of the patient model is known at every moment, which is a simplification as well, since, the real system's input is the ingested food (here, the dosed amount of glucose $(d(t))$ ), not the absorbed glucose from the gastrointestinal system $\left(R_{a}(t)\right)$. The goal of this simplification was to describe more accurate the patient 


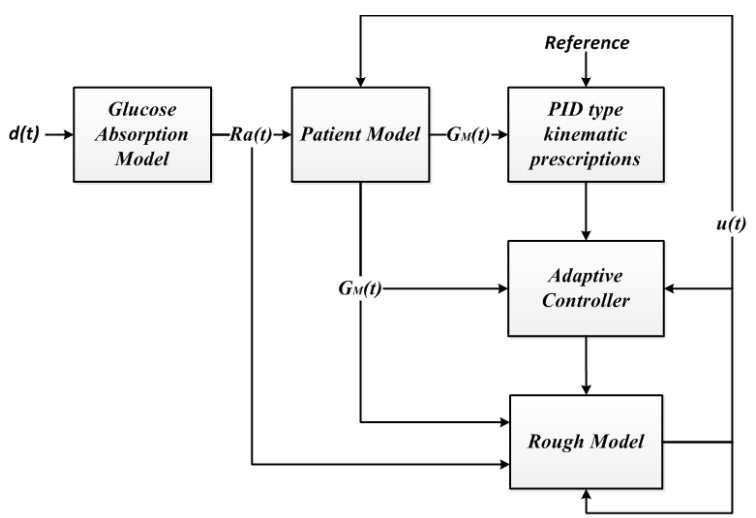

Figure 4. Schematic structure of the control environment

model's dynamics with the rough model, the $d(t)$ also can be used for this purpose.

2) Constraints about the used control scheme: The most advantage of the RFPT-based method, namely, the modelfree approach, it appears as a disadvantage in the accuracy, which is the natural consequence of the fact that the controller does not know anything about the controlled system's inside operation. The applied method to catch the dynamics of the patient model includes inaccuracies, since, we not used the patient model during the designing of the RM. The used second order polynomials from simplification reason is not to accurate and the whole system has other inside dynamical effects, which are not necessarily reflecting in this solution. The adaptive controller is responsible to maintain the sideeffects of the simplifications and inaccuracies through gives information about the required of control signal to reach the control goals. The main constraint with the adaptive controller is the optimization of the control parameters, which requires an individual approach case-by-case and the application will determines it [21].

\section{RESUlts}

We have tested our solution with two glucose intake protocols in the developed in-silico simulation environment with different length. The first glucose intake protocol was the following: $8 \mathrm{am}, 50 \mathrm{~g} ; 13 \mathrm{pm}, 70 \mathrm{~g} ; 20 \mathrm{pm}, 70 \mathrm{~g}$. This sequence is repeating over a week, the total simulation time was 168 hours. The result can be seen on Fig. 5 .

The Fig. 5. shows, that the controller can handle the appearing glucose $\left(R_{a}(t)\right)$ and adapted to the requirements of the system with injecting insulin to reach the prescribed set-point value. The variables started from the steady-state condition and after the initial transient relaxation, because of the recurring input the system showed the expected recurring, oscillating behavior.

The second glucose intake protocol was a randomized one with various intake amounts and time-points. On Fig. 6. can be seen, that after the initial transients, the controller adapting to
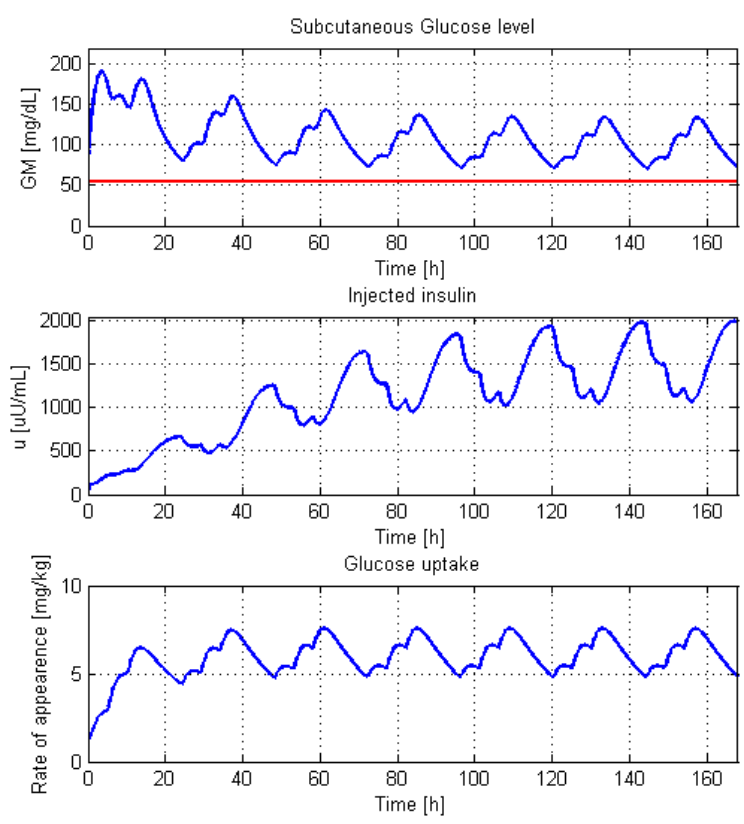

Figure 5. Result of a one week simulation with first feeding protocol [Control parameters: $\Lambda=0.015, A_{c t r l}=1 \exp ^{-} 4, K_{c t r l}=-1000, B_{c t r l}=1$, Set-point $\left(G_{N}\right)=100 \mathrm{mg} / \mathrm{dL}$ ]

the systems needs, however, because of the randomized intake, this adoption is changing all the time, as we expected. The initial values of the simulation were calculated based on 2 , beside $36 \mu \mathrm{U} / \mathrm{mL}(\approx 250 \mathrm{pmol} / \mathrm{L})$, because we would have liked to that the initial conditions not to be optimal. Clearly visible, that the controller can handle the uncertainties like this.

The last figure shows a CVGA result (which is a frequently used evaluating method in diabetes control [22]) of a 53 days long simulation with the following control parameters: $\Lambda=0.0125, A_{c t r l}=1 \exp ^{-3}, K_{c t r l}=-1000, B_{c t r l}=-1$. The randomized intake parameters were: 3 glucose intake at every 24 hours with taking into account that the virtual patient feeding happens during the first 16 hours with minimum 4 hours between the each intakes; the amounts are changing between $40 \mathrm{~g}$ and $70 \mathrm{~g}$, randomly. The results is shown by Fig. 7.

The Fig. 7. shows that the controller can handle this various environment, however, the unfavorable randomized intakes are degrades the adaptivity and producing higher deviation in the daily maximum and minimum of BG levels.

\section{CONCLUSION AND FUTURE WORK}

In this paper, our goal was to proof the usability of the RFPT-based control design method in the field of diabetes control. We investigated several situations and the simulations were encouraging, we presented here three of them. The RFPTbased method is appropriate from different point of views, which were detailed in the text. In our future work, we are going to investigate the design method from different directions, namely, we will examine the possibilities of parameter 

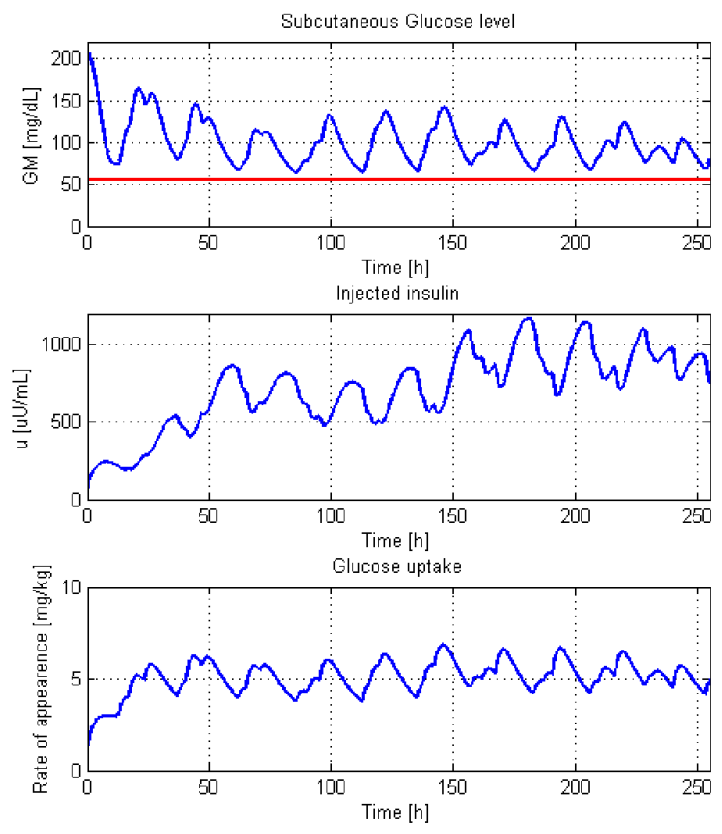

Figure 6. Result of a 255 hours simulation with second feeding protocol [Control parameters: $\Lambda=0.0125, A_{\text {ctrl }}=1 \exp ^{-3}, K_{c t r l}=-1000$, $B_{c t r l}=-1$, Set-point $\left(G_{N}\right)=95 \mathrm{mg} / \mathrm{dL}$ ]

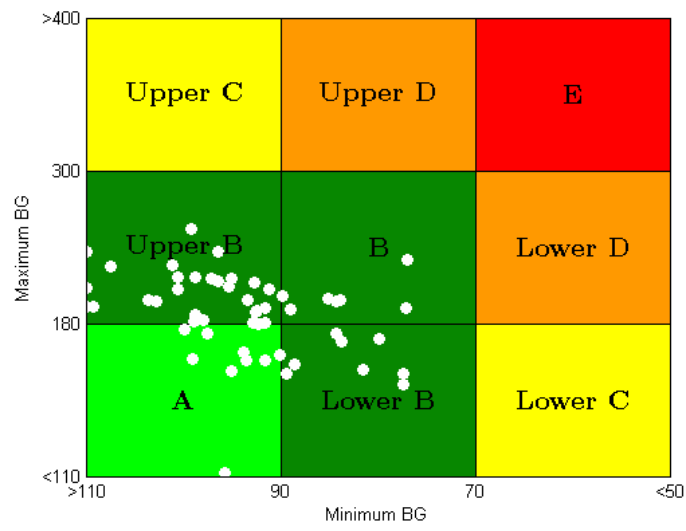

Figure 7. Result of a 255 hours simulation with second feeding protocol [Control parameters: $\Lambda=0.0125, A_{c t r l}=1 \mathrm{exp}^{-3}, K_{c t r l}=-1000$, $B_{c t r l}=-1$, Set-point $\left(G_{N}\right)=95 \mathrm{mg} / \mathrm{dL}$ ]

identification and optimization, control law and adaptivity function selection and other point of views.

\section{ACKNOWLEDGMENT}

The Authors thankfully acknowledge the support by the Research Fund of Óbuda University.

L. Kovács is also supported by the János Bolyai Research Scholarship of the Hungarian Academy of Sciences.

\section{REFERENCES}

[1] ADA, "Diagnosis and classification of diabetes mellitus," Diab Care, vol. $27,2004$.

[2] A. Fonyó and E. Ligeti, Physiology (in Hungarian), 3rd ed. Budapest, Hungary: Medicina, 2008

[3] F. Chee and T. Fernando, Closed-Loop Control of Blood Glucose. Springer, 2007

[4] A. Lyapunov, Stability of motion. Academic Press, New-York and London, 1966.

[5] R. Hovorka, V. Canonico, L. Chassin, U. Haueter, M. Massi-Benedetti, M. Orsini-Federici, T. Pieber, H. Schaller, L. Schaupp, T. Vering, and W. M.E., "Nonlinear model predictive control of glucose concentration in subjects with type 1 diabetes," Physiol Meas, vol. 25, no. 4, pp. 905920, 2004.

[6] P. Herrero, P. Georgiou, N. Oliver, D. Johnston, and C. Toumazou, "A bio-inspired glucose controller based on pancreatic $\beta$-cell physiology," J Diab Sci Technol, vol. 6, no. 3, pp. 606-616, 2012.

[7] E. Atlas, R. Nimri, S. Miller, E. Grunberg, and M. Phillip, "Md-logic anrtificial pancreas system. a pilot study in adults with type 1 diabetes," Diab Care, vol. 33, pp. 1072-1076, 2010.

[8] L. Kovács, B. Benyó, J. Bokor, and Z. Benyó, "Induced L2-norm minimization of glucose-insulin system for type I diabetic patients," Comp Meth Prog Biomed, vol. 102, pp. 105-118, 2011.

[9] P. Szalay, G. Eigner, and L. Kovács, "Linear matrix inequality-based robust controller design for type-1 diabetes model," in IFAC 2014 - 19th World Congress of The International Federation of Automatic Control, Cape Town, South-Africa, 2014, pp. 9247-9252.

[10] J. Bokor and P. Szabó, "Loop shifting and non-conservative qlpv design," ACTA Pol Hung, vol. 11, no. 4, pp. 7-20, 2014.

[11] K. Hosseini-Suny, H. Momeni, and F. Janabi-Sharifi, "Model reference adaptive control design for a teleoperation system with output prediction," J Intell Robot Syst, vol. 59, pp. 319-339, 2010.

[12] T. Várkonyi, J. Tar, and I. Rudas, "Improved stabilization for robust fixed point transformations-based controllers," J Adv Comp Int Inform, vol. 17, no. 3, pp. 418-424, 2013.

[13] J. Tar, J. Bitó, L. Nádai, and J. Machado, "Robust fixed point transformations in adaptive control using local basin of attraction," ACTA Pol Hung, vol. 6, no. 1, pp. 21-37, 2009.

[14] L. Magni, D. Raimondo, L. Bossi, C. Dalla Man, G. De Nicolao, B. Kovatchev, and C. Cobelli, "Model predictive control of type 1 diabetes: An in silico trial," J Diab Sci Techn, vol. 1, pp. 804-812, 2007.

[15] L. Magni, Raimondo, C. Dalla Man, G. De Nicolao, B. Kovatchev, and C. Cobelli, "Model predictive control of glucose concentration in subjects with type 1 diabetes: an in silico trial," in 17th World Congress The International Federation of Automatic Control, Seoul, Korea, 2008, pp. 4246-4251.

[16] C. Dalla Man, R. Rizza, and C. Cobelli, "Mixed meal simulation model of glucose-insulin system," in 28th IEEE EMBC Conference, New York, USA, 2006, pp. 307-310.

[17] _ - "Meal simulation model of glucose-insulin system," JAm J Physiol Endocrinol Metab, vol. 45, no. 12, pp. 1740-1749, 2007.

[18] C. Dalla Man, F. Micheletto, D. Lv, M. Breton, B. Kovatchev, and C. Cobelli, "The uva/padova type 1 diabetes simulator: New features." J Diab Scien Techn, vol. 8, no. 8, pp. 26-34, 2014

[19] K. Kósi, J. Tar, and I. Rudas, "Improvement of the stability of RFPTbased adaptive controllers by observing "precursor oscillations"," In Proc. of the $9^{\text {th }}$ IEEE Intl. Conf. on Computational Cybernetics, Tihany, Hungary, pp. 267-272, 2013.

[20] S. Banach, "Sur les opérations dans les ensembles abstraits et leur application aux équations intégrales (About the Operations in the Abstract Sets and Their Application to Integral Equations)," Fund. Math., vol. 3, pp. 133-181, 1922.

[21] P. Hováth, "Investigation of robust-fixed point transfromation in case of type 1 diabetes control (in hungarian)," Master's thesis, Faculty of Electrical Engineering and Informatics, Budapest University of Technology and Economics, Budapest, Hungary, 2014.

[22] L. Magni, D. Raimondo, C. Dalla Man, M. Breton, S. Patek, G. De Nicolao, C. Cobelli, and B. Kovatchev, "Evaluating the efficacy of closed-loop glucose regulation via control-variability grid analysis," J Diab Scien Techn, vol. 2, no. 4, pp. 630-635, 2008. 